\title{
Molecular characterization and ligand binding specificity of the PDZ domain-containing protein GIPC3 from Schistosoma japonicum
}

\author{
Yi Mu', Haiming Huang ${ }^{1,3}$, Shuai Liu², Pengfei Cai ${ }^{2 *}$ and Youhe Gao ${ }^{1 *}$
}

\begin{abstract}
Background: Schistosomiasis is a serious global health problem that afflicts more than 230 million people in 77 countries. Long-term mass treatments with the only available drug, praziquantel, have caused growing concerns about drug resistance. PSD-95/Dlg/ZO-1 (PDZ) domain-containing proteins are recognized as potential targets for the next generation of drug development. However, the PDZ domain-containing protein family in parasites has largely been unexplored.
\end{abstract}

Methods: We present the molecular characteristics of a PDZ domain-containing protein, GIPC3, from Schistosoma japonicum (SjGIPC3) according to bioinformatics analysis and experimental approaches. The ligand binding specificity of the PDZ domain of SjGIPC3 was confirmed by screening an arbitrary peptide library in yeast two-hybrid $(\mathrm{Y} 2 \mathrm{H})$ assays. The native ligand candidates were predicted by Tailfit software based on the C-terminal binding specificity, and further validated by $\mathrm{Y} 2 \mathrm{H}$ assays.

Results: SjGIPC3 is a single PDZ domain-containing protein comprised of 328 amino acid residues. Structural prediction revealed that a conserved PDZ domain was presented in the middle region of the protein. Phylogenetic analysis revealed that SjGIPC3 and other trematode orthologues clustered into a well-defined cluster but were distinguishable from those of other phyla. Transcriptional analysis by quantitative RT-PCR revealed that the SjGIPC3 gene was relatively highly expressed in the stages within the host, especially in male adult worms. By using $\mathrm{Y} 2 \mathrm{H}$ assays to screen an arbitrary peptide library, we confirmed the C-terminal binding specificity of the SjGIPC3-PDZ domain, which could be deduced as a consensus sequence, -[SDEC]-[STIL]-[HSNQDE]-[VIL]*. Furthermore, six proteins were predicted to be native ligand candidates of SjGIPC3 based on the C-terminal binding properties and other biological information; four of these were confirmed to be potential ligands using the $\mathrm{Y} 2 \mathrm{H}$ system.

Conclusions: In this study, we first characterized a PDZ domain-containing protein GIPC3 in S. japonicum. The SjGIPC3-PDZ domain is able to bind both type I and II ligand C-terminal motifs. The identification of native ligand will help reveal the potential biological function of SjGIPC3. These data will facilitate the identification of novel drug targets against $S$. japonicum infections.

Keywords: Schistosoma japonicum, GIPC3, PDZ domain-containing protein, Ligand binding specificity

\footnotetext{
* Correspondence: puffy_cai@163.com; youhegao@163.com

${ }^{2} \mathrm{MOH}$ Key Laboratory of Systems Biology of Pathogens, Institute of Pathogen

Biology, Chinese Academy of Medical Sciences \& Peking Union Medical

College, Beijing 100730, P.R. China

'National Key Laboratory of Medical Molecular Biology, Dept. of Physiology

and Pathophysiology, School of Basic Medicine, Peking Union Medical

College, Institute of Basic Medical Sciences, Chinese Academy of Medical

Sciences, Beijing 100005, P.R. China

Full list of author information is available at the end of the article
} 


\section{Background}

Schistosomiasis, caused by the parasitic blood fluke of the genus Schistosoma, remains a major global public health problem that affects more than 230 million people living in the endemic areas of 77 countries worldwide (more than 95\% in Africa) (http://www.who.int/ mediacentre/factsheets/fs115/en/index.html). Three major pathogenic schistosome species, Schistosoma mansoni, Schistosoma haematobium, and Schistosoma japonicum, are known to contribute to the disease loads. The treatment of schistosomiasis is based on the long-term mass treatment with the only available drug, praziquantel (PZQ). However, concerns about the problem of drug resistance have necessitated a search for alternative treatments [1-3]. The decoding of the genomes of three major pathogenic blood flukes has provided a valuable entity for the systematic identification of possible drug targets against the parasite [4-6].

Protein-protein interactions (PPIs) offer a realm of less explored potential drug targets, and are recognized to be the novel targets of next-generation drug development [7]. The PDZ domain is one of the most important PPI modules, and is emergently regarded as a novel target of drug discovery $[8,9]$. PDZ domains are built of approximately 70 to 90 residues and form a compact globular fold consisting of a core of six $\beta$-strands $(\beta \mathrm{A}-\beta \mathrm{F})$ and two $\alpha$-helices $(\alpha \mathrm{A}, \alpha \mathrm{B})[10]$. The $\mathrm{C}$-terminus $(4$ to $5 \mathrm{C}$ terminal residues) of partners usually projects to a hydrophobic pocket formed with the carboxylatebinding loop ( $\beta \mathrm{A}-\beta \mathrm{B}$ loop), which contains the conserved "GLGF" motif, and the ?B helix of the PDZ domain [11]. The PDZ binding motifs have been conventionally placed into four classes based on the extreme C-terminus: Class I, $-[\mathrm{S} / \mathrm{T}]-X-\Phi^{*}$; Class II, $-\Phi-X-$ $\Phi^{*}$; Class III, -[D/E/K/R]- $X-\Phi^{*}$; and Class IV, $-X-\psi-[\mathrm{D} / \mathrm{E}]$ "; where $X$ is any amino acid, $\Phi$ is a hydrophobic residue, $\psi$ is an aromatic residue, and " represents the stop codon of the peptide [12]. Although the PDZ domains are conserved in a variety of organisms, no detailed literature about the schistosome-derived PDZ domain has been reported thus far.

As a benefit of the decoding of the genome, several PDZ domain-containing proteins were deposited in the public S. japonicum database. Among them, one is homologous to the mammalian GAIP-interacting proteins, $C$ terminus (GIPCs, also known as TIP-2, GLUT1CBP, RGS19IP1, SEMCAP-1, and synectin); here we have designated the $S$. japonicum homologue as SjGIPC3. GIPCs are characterized by a single, centrally located PDZ domain for ligand binding. GIPC1 was originally found to bind to the $G_{\alpha i}$ GTPase-activating protein RGS-GAIP and have been suggested to exert function in a G-protein coupled complex mediating vesicular trafficking [13]. Accumulating data have confirmed that the
PDZ domain of GIPCs can interact with a panel of partners through $\mathrm{C}$-terminal region recognition. Most of these partners are transmembrane receptors, such as Tax [14], M-semF [15], Glut1 [16], neuropilin-1 [17], Syndecan-4 [18], and so on [19-28]. In addition, human and Drosophila GIPC orthologues can also bind to the non-receptor protein, myosin VI [29,30]. Recently, GIPC orthologues were regarded as novel and potential targets for therapeutic development for the treatment of human cancers [31,32]. Several cell-permeable octapeptides targeting the PDZ domain of GIPCs were designed based on binding specificity and have shown their powers in inhibition of proliferation and induction of apoptosis in some types of cancer [31,33].

In this study, we present for the first time the molecular characteristics of a PDZ-containing protein from $S$. japonicum, GIPC3. We analyzed the expression levels of SjGIPC3 during development of the parasite. To reveal its ligand binding specificity, a random peptide library constructed from human genomic DNA was used in yeast two-hybrid $(\mathrm{Y} 2 \mathrm{H})$ assays to screen for the Cterminal binding motifs of the SjGIPC3-PDZ domain. Furthermore, the potential ligands of SjGIPC3 were predicted by bioinformatic approaches based on the Cterminal binding specificity, and validated in the $\mathrm{Y} 2 \mathrm{H}$ system. This work will facilitate the identification of novel drug targets against $S$. japonicum.

\section{Methods}

\section{Parasites and animals}

The S. japonicum-infected Oncomelania hupensis were purchased from Jiangxi Institute of Parasitic Diseases, Nanchang, China. Cercariae were shed from the infected snails and harvested for total RNA isolation. New Zealand White rabbits were percutaneously infected with cercariae. Hepatic schistosomula and adult worms were obtained from the rabbits by hepatic-portal perfusion at 2 and 6 weeks post-infection, respectively. Male and female adult worms were manually separated with the aid of a light microscope. Eggs were isolated from liver tissues of rabbits after 6 weeks of infection by the sieving and enzymatic digestion method [34].

\section{Bioinformatics analysis of SjGIPC3}

The nucleotide and deduced amino acid sequence of SjGIPC3 were analyzed from NCBI. The PDZ domain of SjGIPC3 protein was predicted in the Conserved Domain Database (CDD) v.2.25 of NCBI (http://www.ncbi. nlm.nih.gov/cdd) [35]. The three-dimensional structure of the SjGIPC3-PDZ domain was predicted by the Rosetta server (http://robetta.bakerlab.org/) [36] with the PDZ domain in GIPC2 (PDB code: 3GGE - chain A) as the template. The $3 \mathrm{D}$ structure was further refined with PyMOL Viewer program (http://www.pymol.org/). 


\section{Multiple sequence alignment and phylogenetic analysis}

The GIPC homologous sequences of other species were retrieved from GenBank using SjGIPC3 as bait. Protein sequences were aligned by ClustalX 2.0. The alignment was refined with GeneDoc software. Phylogenetic analysis was performed as previously described [37]. The alignment was further optimized by deletion of highly variable $\mathrm{N}$ - and $\mathrm{C}$-terminal regions. The phylogenetic tree was constructed with MEGA 4 using the neighborjoining method. Bootstrap values were expressed as percentage of 1000 replicates.

\section{Quantitative RT-PCR}

Total RNAs of S. japonicum at different developmental stages (cercariae, hepatic schistosomula, separated adult male and female worms, and eggs) were extracted using Trizol reagent (Invitrogen). The contaminating genomic DNA was removed from RNA samples with TURBO DNA-free ${ }^{\mathrm{TM}}$ kit (Ambion, CA, USA). RNA quantification and quality control was conducted by $1 \%$ agarose gel electrophoresis and Nanodrop ND-1000 spectrophotometer (Nanodrop Technologies, Wilmington, DE). For each sample, $1 \mu \mathrm{g}$ total RNA was reverse transcribed into first-strand cDNA using SuperScript ${ }^{\mathrm{TM}}$ III Reverse Transcriptase Kit (Invitrogen), with Oligo dT (15) primer. cDNA synthesis was performed as follows: $25^{\circ} \mathrm{C}$ for $5 \mathrm{~min}, 50^{\circ} \mathrm{C}$ for $1 \mathrm{~h}, 70^{\circ} \mathrm{C}$ for $15 \mathrm{~min}$. Each PCR reaction contained $12.5 \mu \mathrm{l}$ of $2 \times$ Brilliant II SYBR Green QPCR Master Mix (Agilent, USA), $1 \mu$ l diluted cDNA (20×), 1 $\mu \mathrm{l}$ of the forward and reverse primer pair (final concentration: $0.2 \mu \mathrm{M}$ each primer, Additional file 1 Table S1), and $10.5 \mu \mathrm{l}$ of sterile water. The PCR program included 40 cycles with denaturation at $95^{\circ} \mathrm{C}$ for $30 \mathrm{~s}$, followed by annealing and extension at $60^{\circ} \mathrm{C}$ for $1 \mathrm{~min}$. Quantification of the relative differential expression for SjGIPC3 gene was performed by normalizing against the PSMD4 transcript (26S proteasome non-ATPase regulatory subunit 4, GenBank accession number: FN320595) and applying the comparative $2^{-\Delta \Delta C t}$ method, according to the SDS 1.4 software (Applied Biosystems, Foster City, USA) [38].

\section{Construction of bait plasmids}

The DNA fragments encoding full-length SjGIPC3 and the PDZ domain region (AA 99-195) were amplified from the adult worm cDNA templates using high fidelity Phusion DNA polymerase (Finnzymes Oy, Finland) (Primer sets are listed in Additional file 1 Table S1). The PCR was performed with an initial denaturation for 1 min at $98^{\circ} \mathrm{C}$. Thirty PCR cycles were performed as follows: $98^{\circ} \mathrm{C}$ for $5 \mathrm{~s}, 50^{\circ} \mathrm{C}$ for $30 \mathrm{~s}$, and $72^{\circ} \mathrm{C}$ for $15 \mathrm{~s}$. The final extension was $5 \mathrm{~min}$ at $72^{\circ} \mathrm{C}$. The amplicons were digested with EcoRI and BamHI, and cloned into GAL4 $\mathrm{BD}$ vector, pBridge. The recombinant plasmids were transformed into DH5 $\alpha$ (DE3) Escherichia coli and positive clones were selected for sequencing.

\section{Yeast two-hybrid screening of random peptide library}

Each GAL4 BD-fusion bait plasmid was transformed into the yeast strain CG1945 using the lithium acetate procedure. The transformants were grown on $\mathrm{SD} /$-Trp plates and lacZ assays were performed to examine selfactivation. The transformants were further spread on $\mathrm{SD} /$-His/-Trp plates with different concentration of 3amino-1,2,4-triazole for leakage test. The random peptide library (constructed with Tsp509I-digested human genomic DNA fragments) [39] was screened following the MATCHMAKER Two-Hybrid System protocol (Clontech). The transformants were screened on $\mathrm{SD} /$ Trp/-His/-Leu plates with different concentrations of 3amino-1,2,4-triazole (2.5 $\mathrm{mM}$ for full-length SjGIPC3 bait transformants, and $20 \mathrm{mM}$ for SjGIPC3-PDZ bait transformants) and further verified by the improved LacZ assays. After rescue, the potential positive plasmids were isolated and retransformed into the yeast strain CG1945 containing corresponding bait plasmid. Only the clones that were positive for all the reporter assays and confirmed by at least two independent tests were selected for specific interactions and sequenced [40].

\section{Prediction of candidate ligands}

A consensus C-terminal binding sequence of SjGIPC3PDZ domain was deduced from sequence alignment of positive clones from yeast two-hybrid screening. The $S$. japonicum predicted protein sequences were downloaded from SDSPB (http://lifecenter.sgst.cn/schistosoma/cn/schdownload.do). The Tailfit software [41] was used to search against the $S$. japonicum protein sequences to retrieve potential ligand proteins whose carboxyl termini matched the consensus-binding sequence. These peptide sequences were then manually blasted against NCBI's non-redundant protein database to filter the truncated fragments lacking of the Cterminus. The most promising candidate ligand proteins were selected further based on biological information such as subcellular localization and potential molecular function.

\section{Confirmation of candidate SjGIPC3-ligand interactions}

The GAL4 AD plasmids expressing the carboxylterminus of the candidate ligands of the SjGIPC3-PDZ domain were first constructed. The self primer template PCR was performed to produce DNA fragments encoding the 10-11 amino acid residues of extreme carboxyl-terminus of potential ligands (The primer sets were shown in Additional file 1 Table S1). The PCR procedure included thirty cycles as follows: $98^{\circ} \mathrm{C}$ for 5 $\mathrm{s}, 50^{\circ} \mathrm{C}$ for $25 \mathrm{~s}$, and $72^{\circ} \mathrm{C}$ for $1 \mathrm{~s}$, and a final 
extension at $72^{\circ} \mathrm{C}$ for $2 \mathrm{~min}$. The resulting DNA fragments were digested with EcoRI and BamHI endonucleases, and cloned into the EcoRI/BamHI sites of GAL4 $\mathrm{AD}$ vector, pGADT7 followed by sequencing. Each constructed plasmid was co-transformed with the SjGIPC3PDZ domain or full-length SjGIPC3 bait plasmid into yeast strain CG1945, respectively. The positive interactions were selected in the yeast two-hybrid system as mentioned above.

\section{Results}

\section{Molecular characteristics of S. japonicum GIPC3}

By searching the $S$. japonicum proteomic database, we found that at least $25 \mathrm{PDZ}$ domains are distributed among 16 proteins. S. japonicum GIPC3 is a single PDZ domain-containing protein, which is 328 amino acids in length, with a theoretical molecular weight of $37 \mathrm{kDa}$. By performing a CD-search, we found that the PDZ domain was located in the middle region of SjGIPC3 protein. The peptide sequence of SjGIPC3 was aligned with sequences of orthologues from other taxa. The GIPC homology 1 (GH1) and PDZ domains of GIPC proteins were relatively well conserved, in contrast to the GIPC homology 2 (GH2) domain and the flanking $\mathrm{N}$ - and $\mathrm{C}$ terminal regions (Figure 1). Homology analysis revealed that SjGIPC3 shares a relatively high degree of sequence homology with S. mansoni orthologue (GenBank Accession number: XP_002569948, identity $=78 \%$, only aa1894-2197 region was used for calculating identity) and followed by share $46 \%$ identity with Clonorchis sinensis orthologue (GenBank Accession number: GAA51139). The sequence in the conserved "GLGF" motif is replaced by "SFGL" in SjGIPC3, which is different to that in any other GIPC orthologues (Figure 1). Structural prediction revealed that the SjGIPC3-PDZ domain was comprised of two $\alpha$-helixes and five $\beta$-sheets (lacking $\beta E$, Figure 2A), with a hydrophobic pocket formed between $\beta B$ and $\alpha B$, in which the residue $F$ from the unique "GLGF" motif ("SFGL" in the SjGIPC3-PDZ domain, while "ALGL" or "SLGL" in the PDZ domains of mammalian GIPCs) may affect the ligand binding specificity of the PDZ domain (Figure 2B).

\section{Phylogenetic analysis of SjGIPC3}

The sequences of GIPC orthologues from other taxa were retrieved to create a phylogenetic tree using the Neigh-bor-Joining method. SjGIPC3 was clustered with S. mansoni and C. sinensis orthologues to form a clade, which was divergent from those of other phyla, even with the parasitic nematode-derived orthologue, AsGIPC1 (Figure 3). However, less is known about whether this divergence of fluke GIPC orthologues is related to adaptations specific to their parasitic life.

\section{Transcriptional analysis of the SjGIPC3 gene at different developmental stages of the parasite}

To determine the transcriptional patterns of SjGIPC3 at different developmental stages and between sexes, qRTPCR was performed using an optimal reference gene, 26S proteasome non-ATPase regulatory subunit 4 (PSMD4), as an internal control [42]. As a result, we observed that the SjGIPC3 gene was ubiquitously expressed at different developmental stages, but in a stage-biased pattern. The expression level of the SjGIPC3 gene was lower in the cercarial stage than in any other stages in which it was detected, suggesting that the function of SjGIPC3 is mainly exerted in the stages within the host. In adult worms, the transcriptional level of SjGIPC3 was significantly higher in male than in female worms (Figure 4).

Determination of the ligand binding specificity of SjGIPC3 To investigate the binding properties of the PDZ domain of SjGIPC3, we screened an arbitrary peptide library using $\mathrm{Y} 2 \mathrm{H}$ assays. When the library was screened against the SjGIPC3-PDZ domain, 37 positive clones were sequenced, each encoding a unique carboxy-terminus. Among them, 17 belong to Class I PDZ binding motifs, 16 belong to Class II PDZ binding motifs, and four are unclassified (Table 1). When the library was screened against the full-length SjGIPC3, 42 positive clones were obtained. Sequence analysis revealed that three pairs of clones were identical; thus a total of 39 unique carboxy-terminal sequences were obtained. Among them, 24 belong to Class I PDZ binding motifs, 12 belong to Class II PDZ binding motifs, and three are unclassified (Table 1).

In the first assay using the SjGIPC3-PDZ domain as bait, the domain showed predominant preference for hydrophobic amino acids at the extreme carboxyl terminus $\left(\mathrm{P}^{0}\right)$, especially Leu $(37.8 \%)$, Ile $(32.4 \%)$, or Val (18.9\%). The $\mathrm{P}^{-1}$ of the carboxyl terminus slightly prioritizes polar amino acids, such as Ser (16.2\%), Asn (10.8\%), or Gln (10.8\%), and acidic amino acids, such as Asp $(13.5 \%)$ or Glu (13.5\%). At $\mathrm{P}^{-2}$, the polar amino acids Ser (37.8\%) and Thr (10.8\%) and the aliphatic amino acids Ile (21.6\%) and Leu (18.9\%) were preferentially selected. At $\mathrm{P}^{-3}$, Ser (13.5\%), Asp (18.9\%), and Glu (24.3\%) were preferred, along with the rare occurrence of other amino acids (Figure 5A). Based on the statistical analysis of unique carboxy-terminal sequences, a consensus-binding sequence can be deduced as - $[\mathrm{S} / \mathrm{D} /$ E]-[S/T/I/L]-[S/N/Q/D/E]-[V/I/L]**

In the second assay, full-length SjGIPC3 was probed to screen the random peptide library, and a more significant binding specificity emerged. As in the first assay, hydrophobic amino acids, especially Ile (42.5\%), Leu (21.3\%), or Val (14.2\%), were predominantly preferred at $\mathrm{P}^{0}$, with a slight bias toward the aromatic amino acid Phe (9.5\%). At $\mathrm{P}^{-1}$, the 
Sj GIPC3 Sm GIPC3 Cs GIPC1 Xt GIPC3 Hs GIPC 3 Xt GIPC1 Hs GIPC1 $X+$ GIPC2 Hs GIPC2 Dm Kermit As GIPC1 Ce GIPC1

SjGIPC3 Sm GIPC3

Cs GIPC1

$\mathrm{xt}$ GIPC 3

Hs GIPC 3

Xt GIPC1

Hs GIPC1

$\mathrm{Xt}$ GIPC2

Hs GIPC2

Dm Kermit

As GIPC1

Ce GIPC1

Sj GIPC3

Sm GIPC3

Cs GIPC1

Xt GIPC3

Hs GIPC 3

Xt GIPC1

Hs GIPC1

$X t$ GIPC2

Hs GIPC2

Dm Kermit

As GIPC1

Ce GIPC1

Sj GIPC3

SmGIPC3

Cs GIPC1

$\mathrm{XtGIPC} 3$

HS GIPC 3

Xt GIPC1

Hs GIPC1

$X$ EIPC2

Hs GIPC2

Dm Kermit

As GIPC1

Ce GIPC1

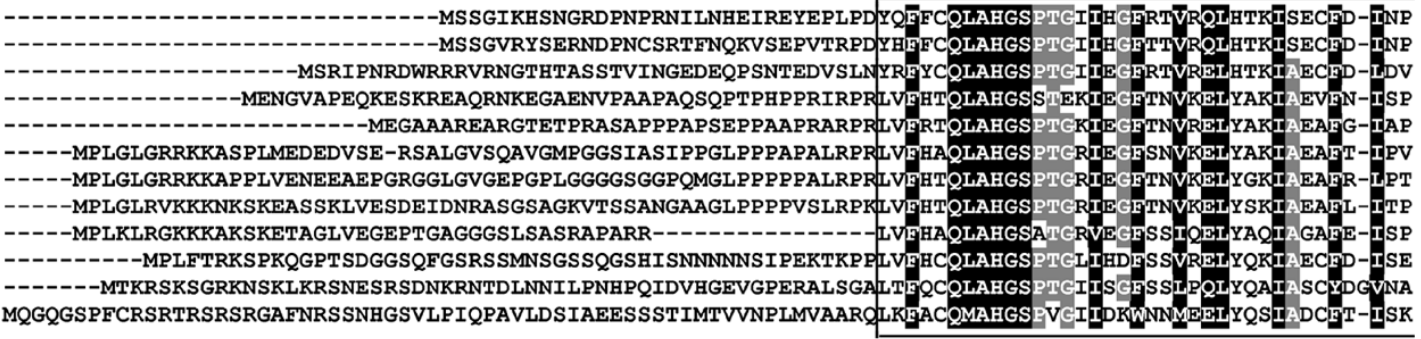

GLGF motif

PDZ domain

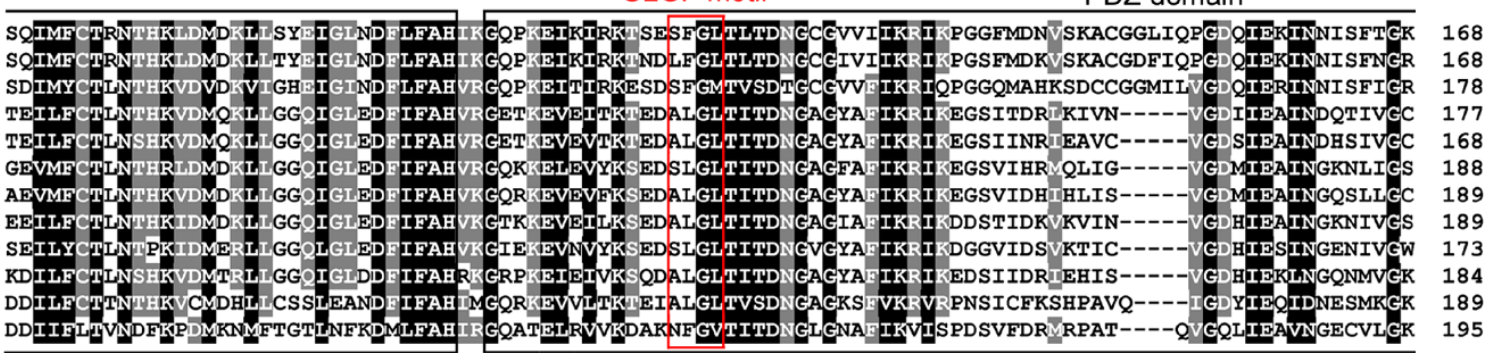

$\mathrm{GH} 2$ domain

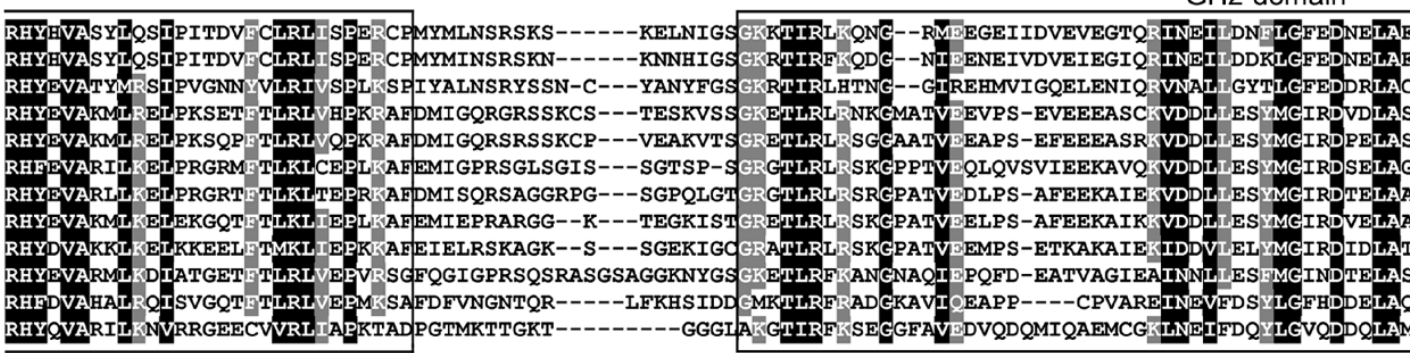

260

260

Figure 1 Sequence alignment of peptide sequences of SjGIPC3 and GIPC orthologues derived from other species with ClustalX 2.0. Black indicates identity in all variants; grey indicates a conservative substitution in at least one variant; white indicates a non-conservative residue; dashes indicate missing residues. GenBank Accession Numbers: SjGIPC3, Schistosoma japonicum, (AAW24514.1); SmGIPC3, Schistosoma mansoni, (XP_002569948); CsGIPC1, Clonorchis sinensis, (GAA51139); XtGIPC3, Xenopus tropicalis, (NP_001096502); HsGIPC3, Homo sapiens, (NP_573568); XtGIPC1, Xenopus tropicalis, (NP_001011331); HsGIPC1, Homo sapiens, (NP_005707); XtGIPC2, Xenopus tropicalis, (NP_001120269); HsGIPC2, Homo sapiens, (NP_060125); DmKermit, Drosophila melanogaster, (NP_652028); AsGIPC1, Ascaris suum, (ADY44129); CeGIPC1, Caenorhabditis elegans, (NP_498017). The GH1, GH2, and PDZ domains are marked with black boxes and the boundaries for these domains were defined based on the previous study [28]. The "GLGF" motif region within the PDZ domains of GIPC orthologues is highlighted by the red box.

preference for Glu (45.2\%) was dramatically increased when compared with the first assay, along with a rare selection of other residues: His (11.9), Ser (11.9\%), Asn (11.9\%), or Asp (9.5\%). The $\mathrm{P}^{-2}$ position demonstrated a dramatic preference for the polar amino acid Ser (54.8\%) and the hydrophobic amino acid Ile (21.4\%); other amino acids, such as
Thr (11.9\%), Val (7.1\%), or Leu (4.8\%), also occurred at a low rate. At $\mathrm{P}^{-3}$, Glu (45.2\%) was predominantly selected, followed by Cys (19.0\%) and Asp (14.3\%) (Figure 5B). Similar to the first assay, a consensus-binding sequence can be inferred from the second $\mathrm{Y} 2 \mathrm{H}$ assay: -[D/E/C]-[S/T/I]-[H/ $\mathrm{S} / \mathrm{N} / \mathrm{D} / \mathrm{E}]-[\mathrm{V} / \mathrm{I} / \mathrm{L}]^{*}$. Finally, based on both assays, the 

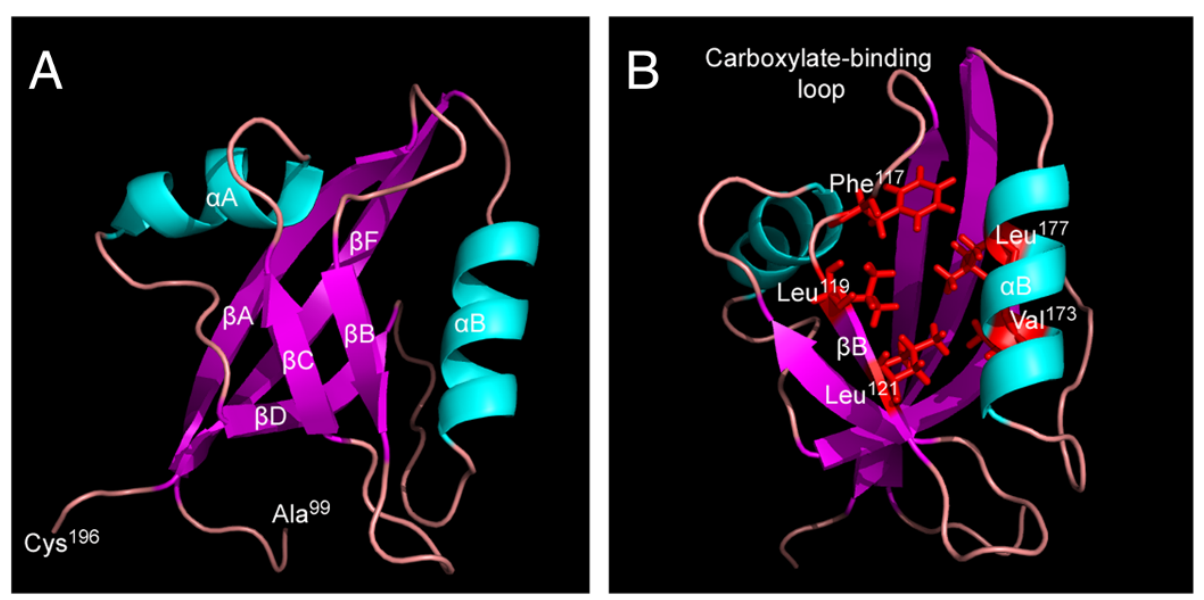

Figure 2 The cartoon diagrams for the 3D structure of the SjGIPC3-PDZ domain. A. The SjGIPC3-PDZ domain is colored by secondarystructure elements (a-helices, cyan; $\beta$-strands, magenta; loops, salmon). B. A hydrophobic pocket was formed by the aB helix, the $\beta B$ strand, and the carboxylate-binding loop. The hydrophobic amino acids within the pocket are highlighted in red. The residue $\mathbf{F}$ in the unique "GLGF" motif (here is "SFGL") may affect the selection of the C-terminal tails of its partner proteins.

consensus-binding sequence of the SjGIPC3-PDZ domain could be reduced to -[SDEC]-[STIL]-[HSNQDE]-[VIL]*, which fits in both class I and class II binding motifs.

\section{Prediction and identification of native ligands of SjGIPC3}

We searched for the potential native ligands of SjGIPC3 in the S. japonicum predicted proteomic database (sjr2_protein. fasta) with the Tailfit program using the consensus-binding sequence -[SDEC]-[STIL]-[HSNQDE]-[VIL]" as bait; 92 proteins that physically fit the consensus binding sequence were fished out. We also performed BLAST searching on NCBI within taxa 6182 directly; using each unique carboxyterminal sequence (the extreme four amino acids) obtained from both $\mathrm{Y} 2 \mathrm{H}$ assays as bait. A panel of proteins whose C-

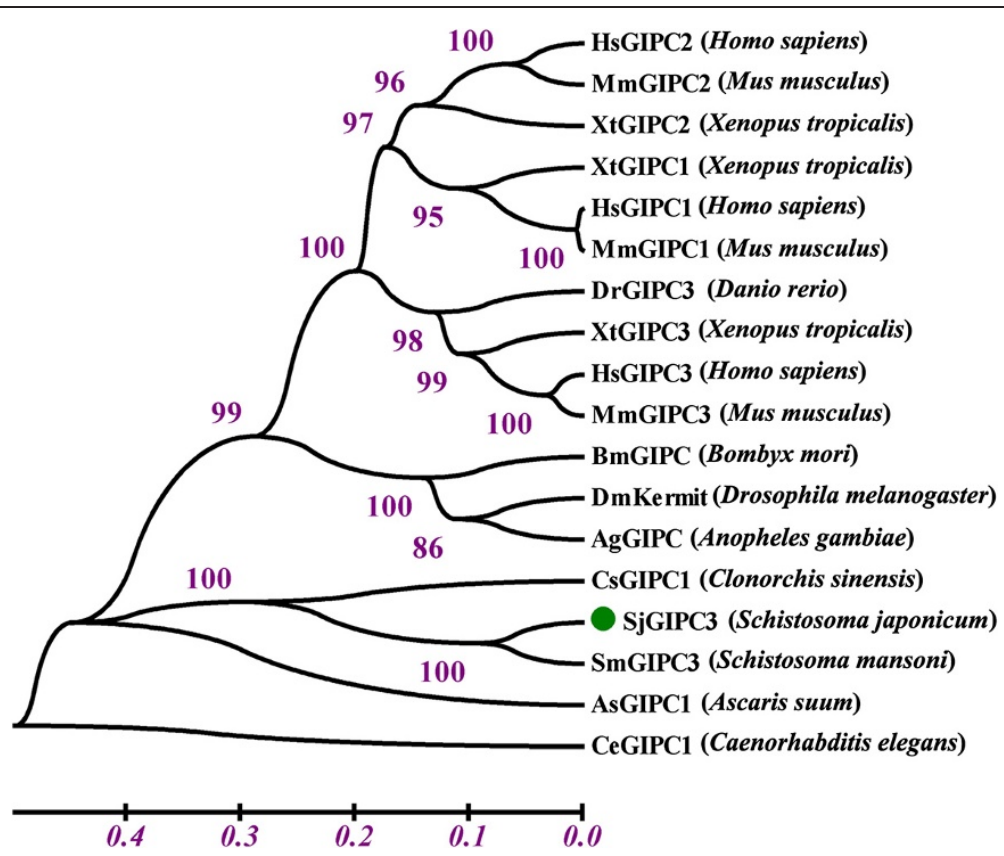

Figure 3 Phylogenetic tree of SjGIPC3 and its orthologues derived from other species. Bootstrap percentages are indicated at each branch. GenBank Accession Numbers: HsGIPC2 (NP_060125), MmGIPC2 (NP_058563), XtGIPC2 (NP_001120269), XtGIPC1 (NP_001011331), HsGIPC1 (NP_005707), MmGIPC1 (NP_061241), DrGIPC3 (CAX13825), XtGIPC3 (NP_001096502), HsGIPC3 (NP_573568), MmGIPC3 (NP_683753), BmGIPC (NP_001040127), DmKermit (NP_652028), AgGIPC (XP_308197) CsGIPC1 (GAA51139), SjGIPC3 (AAW24514.1), SmGIPC3 (XP_002569948), AsGIPC1 (ADY44129), CeGIPC1 (NP_498017). 


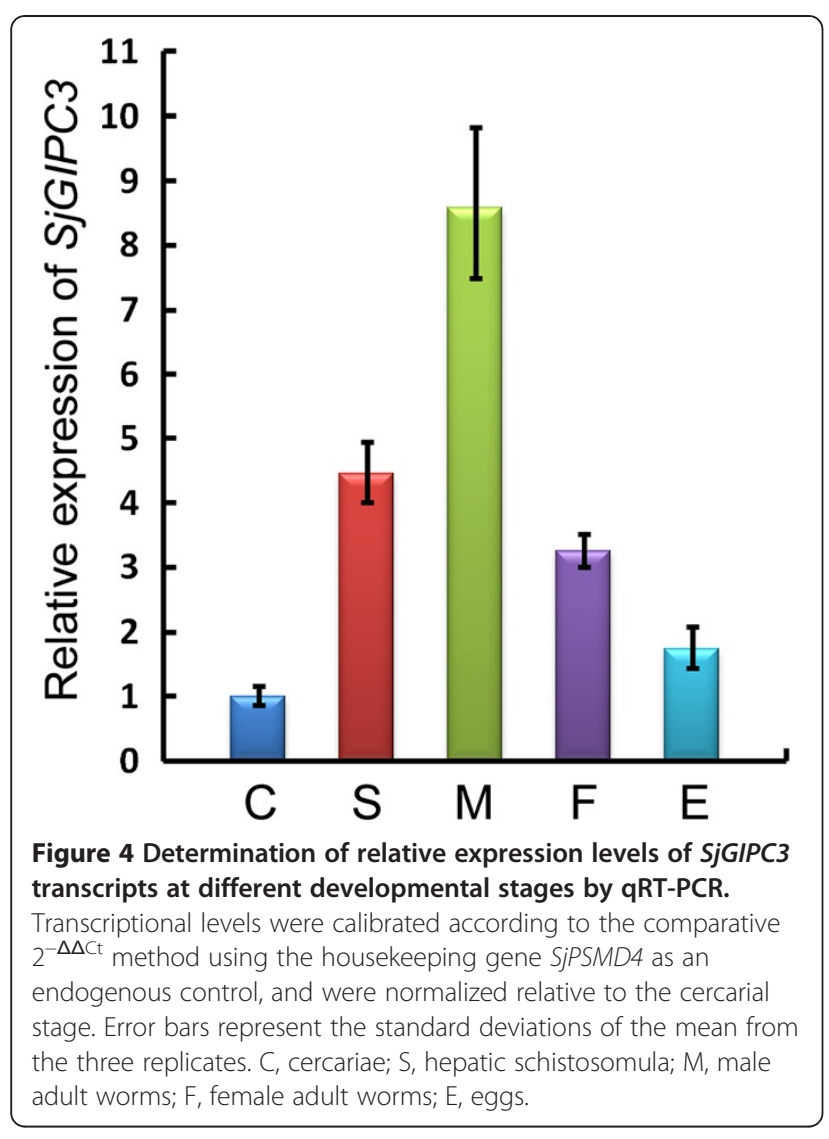

terminal tail matched the unique sequences was retrieved. The protein sequences retrieved from both bioinformatics pipelines were used to perform BLASTP searches on NCBI to manually filter those peptides lacking the C-terminus. We also used integrated biological information, such as transmembrane region prediction, potential molecular functions, and the C-terminal sequence consensus between $S$. japonicum and S. mansoni to determine the most promising candidate ligands. Finally, six proteins were selected as ligand candidates of SjGIPC3 (Table 2). Among them, four were further confirmed to be potential ligands of SjGIPC3 in the $\mathrm{Y} 2 \mathrm{H}$ system.

\section{Discussion}

Domain loss events are extensively widespread in $S$. japonicum, which serves as a notable consequence of the adoption of parasitism [4]. It is estimated that 1,000 protein domains have been abandoned by $S$. japonicum during long-term evolution, which suggests that the remaining domains are necessary for parasitic growth, development, and maturation. The proteomic information revealed that at least 25 PDZ domains are distributed among $16 \mathrm{~S}$. japonicum proteins, suggesting that the PDZ domain is one of the important modules reserved in the parasite to mediate multiple biological processes. In the present study, we have characterized one of the
PDZ domain-containing proteins from $S$. japonicum, GIPC3, for the first time. The ligand binding properties of the SjGIPC3-PDZ domain were determined by $\mathrm{Y} 2 \mathrm{H}$ assays, and its potential ligands were identified.

Screening a highly diverse peptide library provided an opportunity to fish out the potential binding property of SjGIPC3. Whether the SjGIPC3-PDZ domain or fulllength SjGIPC3 were used as bait, analogous binding specificities can be deduced from the unique $\mathrm{C}$-terminal tails obtained from both $\mathrm{Y} 2 \mathrm{H}$ assays. However, the $\mathrm{GH} 1$ and GH2 domains of SjGIPC3 may still affect the binding specificity of the PDZ domain to some degree, because the ligand motifs were more canonical when full-length SjGIPC3 was used as bait. For instance, at $\mathrm{P}^{-1}$, the acidic amino acid Glu was more preferentially selected in the second assay $(45.2 \%)$ than in the first assay (13.5\%). Similarly, at $\mathrm{P}^{-3}, 16$ types of amino acids emerged in the first assay, while only eight kinds of amino acids were presented in the second assay. Also at this position, the occurrence of Glu was $50.0 \%$ when screened against the full-length SjGIPC3, twice the occurrence rate as when the SjGIPC3-PDZ domain was used as bait. Thus, the binding specificity screened using full-length SjGIPC3 as bait may be more authentic to its native status.

Previously, GIPCs have been shown to interact specifically with Class I $\left(-X-\mathrm{S} / \mathrm{T}-X-\Phi^{*}\right)$, II $\left(-\Phi-X-\Phi^{*}\right)$, and III $\left(-X-X-C^{*}\right)$ binding motifs [43]. Moreover, Kermit, the GIPC orthologue in Drosophila, and GLUT1CBP each interact with Myosin VI through an internal sequence within the cargo binding domain $[43,44]$. In this study, we focused on the C-terminal binding specificity of SjGIPC3 and found that the PDZ domain of SjGIPC3 preferred to bind to Class I and II motifs, consistent with the extensive ligand-binding ability of GIPC orthologues in other species [43]. However, regarding the binding of Class II motifs, the only example presented thus far is that the PDZ domain of synectin binds to sydecan-4 through the "-EFYA" motif, in which the aromatic amino acid Phe was selected at $\mathrm{P}^{-2}$ [18]. In our study, it is worth noting that this site can be occupied by the aliphatic amino acid residues Ile and Leu, in addition to Ser and Thr (Table 1). Moreover, in a previous report, the binding motifs of GIPC orthologues trend toward selecting a Val, Ala, or Cys residue at $\mathrm{p}^{0}$ [43]; here, the more hydrophobic amino acids, such as Ile and Leu, were predominantly preferred. These diverse binding characteristics could be utilized for drug development against SjGIPC3.

On average, one PDZ protein is capable of interacting with 17 partners [45]. Although a panel of S. japonicum proteins deposited in the public database bears a C-terminal tail fittting in the consensus binding sequence, the bioinformatic hints at molecular function and localization have prevented them from being ligand candidates of SjGIPC3. Using the 
Table 1 The unique ligand carboxyl termini obtained from Y2H assays using the SjGIPC3-PDZ domain or full-length SjGIPC3 as bait

\begin{tabular}{|c|c|c|c|c|c|}
\hline \multicolumn{3}{|c|}{ The SjGIPC3-PDZ domain as bait } & \multicolumn{3}{|c|}{ Full-length SjGIPC3 as bait } \\
\hline Class I & Class II & Unclassified & Class I & Class II & Unclassified \\
\hline -YTEGETSL* & -TCTLELK|* & -FMVMDSQC* & -LFYKDSEI* & - VFLDEISI* & $\overline{-T K L S E S Q C^{*}}$ \\
\hline$-Y P L F S S H L^{*}$ & $-K L G N F L D I^{*}$ & -CCWMECSV* & $-Y L Y S C S E I^{*}$ & -GNSTEINI* & -KDSQESHC* \\
\hline- KGKECSSI* & $-K L G N S I D V^{*}$ & -PLASDCEV* & $-\mathrm{KLGNCSH}{ }^{*}$ & -DNLEDIEI* & $-A L W L D L D C^{*}$ \\
\hline -AGHSGSCL* & $-E S P F Y L N I^{*}$ & - LIITINLY* & $-C S W L C S D V^{*}$ & -ACDSEVSL* & \\
\hline -FRYQQTDI* & $-\mathrm{VIKLEINL*}$ & & -LSASESEV* & -VMNNTVEF* & \\
\hline -DFCKKSEI* & -LHTNDISV* & & -REVTCSEI* & -PEPWCITL* & \\
\hline -GNSSFSVL* & -RWENYIEI* & & -LWLGESNV* & $-F K K L E I D L^{*}$ & \\
\hline -DSFSTTTL* & -PEAGELLL* & & - KLGNCSEl* & -DRYRDIEI* & \\
\hline -IGNSDTQL* & -NMSDNLDL* & & - RNHVESNI* & -TLNEDIEF* & \\
\hline -STISMSSI* & -LGNYDISL* & & -VSFSETTL* & -FLICEIEI* & \\
\hline- GATDASHI* & -YYSSEVQ|* & & -LLLNETNI* & -EWPLSISF* & \\
\hline -FCKVDSDV* & -GNFSEINI* & & -LGNFETEV* & -NWQLDLEV* & \\
\hline -SDFYSSVL* & -LLENSINL* & & -|CFYSSE|* & & \\
\hline -LCGNRSQV* & - LSFLCIEI $^{*}$ & & -KEQQESDL* & & \\
\hline -DICWESYV* & -HCSEDLIF* & & -FSPRETNL* & & \\
\hline -ARCGSSCL* & -EEESELEM ${ }^{*}$ & & -NSDKPSHT* & & \\
\hline \multirow[t]{8}{*}{-LTQSHSML* } & & & -QQHNESEL* & & \\
\hline & & & -HNSNESSF* & & \\
\hline & & & -GRINESAl* & & \\
\hline & & & - LGNLESHI ${ }^{*}$ & & \\
\hline & & & -EGQGCSEL* & & \\
\hline & & & -KLGNYSEl* & & \\
\hline & & & -GASSVSEV* & & \\
\hline & & & -VFLDETHI* & & \\
\hline
\end{tabular}

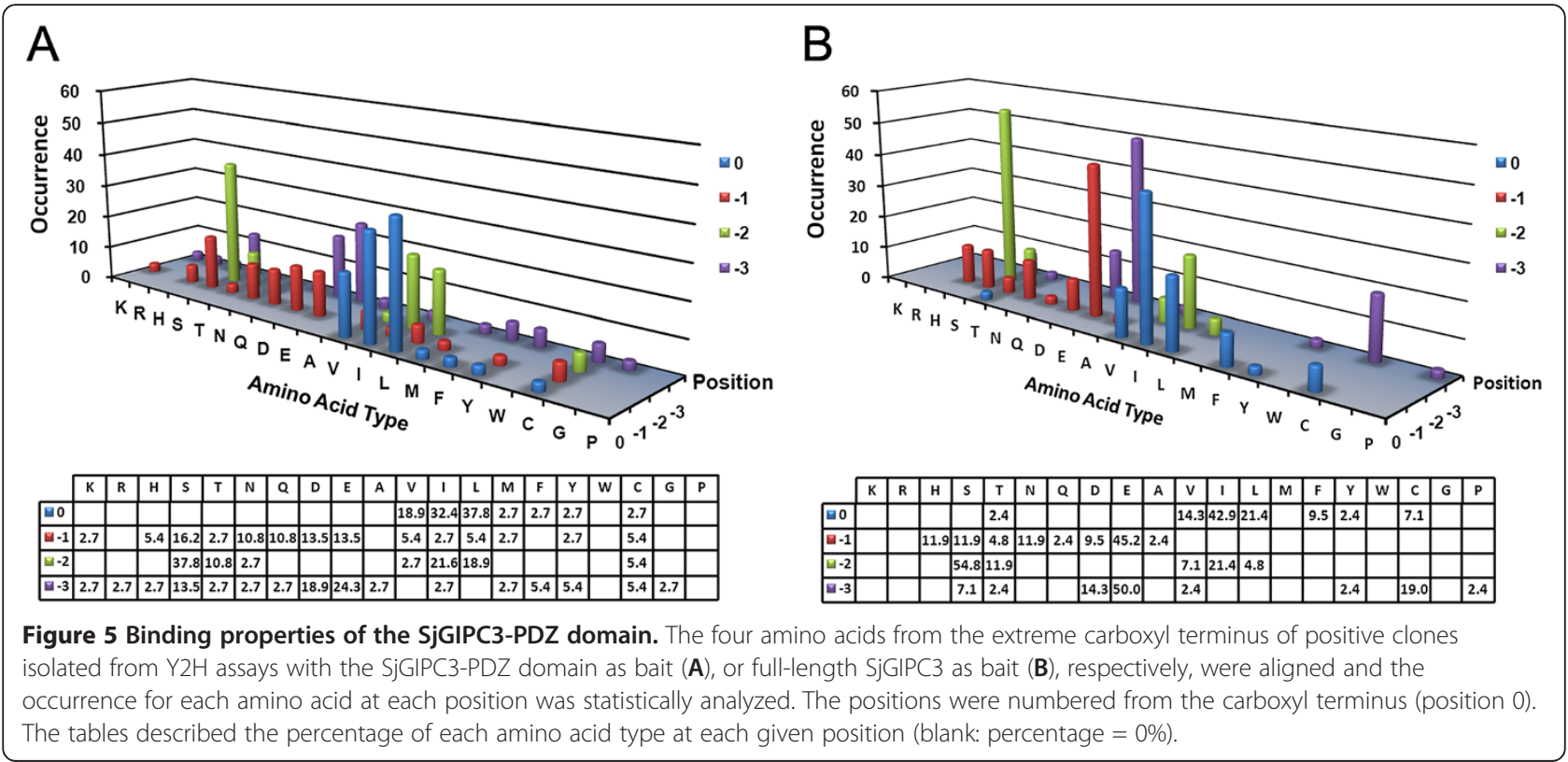


Table 2 Validation of the predicted ligand candidates of SjGIPC3 in the Y2H system

\begin{tabular}{|c|c|c|c|c|c|c|c|}
\hline Bait Sequences & Code & ID & $\begin{array}{l}\text { C-terminus } \\
\text { (S. japonicum) }\end{array}$ & $\begin{array}{l}\text { C-terminus } \\
\text { (S. mansoni) }\end{array}$ & Protein Description & Results $^{3}$ & Results $^{4}$ \\
\hline \multirow[t]{2}{*}{ C-terminal tails $^{1}$} & L1 & ACE06921 & -PDERTTTL* & -HNERTTTL* & glutamate receptor NMDA & $\sqrt{ }$ & $\sqrt{ }$ \\
\hline & L2 & AAX27986 & -PNHLCSDV* & -SNYLCSDV* & hypothetical protein & $\sqrt{ }$ & $\sqrt{ }$ \\
\hline \multirow[t]{4}{*}{-[SDEC]-[STIL]-[HSNQDE]-[VIL] ${ }^{* 2}$} & L3 & CPRT0000005506 & -DESNSLHV* & -EDGNSLSA* & tetraspanin (Sj25) & $x$ & $x$ \\
\hline & $\llcorner 4$ & CPRT0000006203 & -DDRLCSQV* & -DDRLCSQV* & hypothetical protein & $\sqrt{ }$ & $\sqrt{ }$ \\
\hline & L5 & CPRT0000007433 & -ELLTSTEV* & -ELLTSTEV* & Asparagine-rich protein & $\sqrt{ }$ & $\sqrt{ }$ \\
\hline & L6 & CPRT0000008390 & $-K S N V S L S V^{*}$ & $-K S N V S L S V^{*}$ & putative rhodopsin-like orphan GPCR & $x$ & $x$ \\
\hline
\end{tabular}

${ }^{1}:$ The extreme four amino acids of each unique ligand carboxyl terminus listed in Table 1 were used to BLAST on NCBI to retrieve potential ligands within taxa 6182 (S. japonicum).

2: The Tailfit software was used to search against the S. japonicum proteomic database to retrieve potential ligands with the consensus-binding sequence;

3: The validation results from $\mathrm{Y} 2 \mathrm{H}$ assays, using the SjGIPC3-PDZ domain as bait.

4: The validation results from $\mathrm{Y} 2 \mathrm{H}$ assays, using full-length $\mathrm{SjGIPC3}$ as bait.

$\sqrt{ }$ : positive in the $\mathrm{Y} 2 \mathrm{H}$ system; $\mathrm{X}$ : negative in the $\mathrm{Y} 2 \mathrm{H}$ system.

$\mathrm{Y} 2 \mathrm{H}$ assay, four proteins were determined to be potential ligand candidates for SjGIPC3, which suggests that some other potential ligands may not have been discovered yet, likely restricted by the quality of the proteomic database, as truncated fragments have been extensively deposited in the S. japonicum proteomic database.

To adapt to the complexity of the life cycle, schistosomes are equipped with a complex nervous system to sense environmental signals [46]. Transcriptional analysis has revealed that the expression of SjGIPC3 was dramatically up-regulated after invasion of the host, suggesting that it may play an important role (e.g., signal transduction) in the context of the parasite-host interaction. More importantly, the preferential expression of SjGIPC3 in male rather than female adult worms further hinted that the potential function of the protein was related to the processing of environmental information, as male adult worms interface with the host and female adult worms resided in the gynecophoral canal, more stimulating signal would be received by male adult worms [47]. GIPC3 mutations have recently been reported to cause autosomal recessive nonsyndromic sensorineural hearing loss [48-50]. Previously, Yi and his colleagues suggested that the GIPC orthologue in mouse might associate with extrasynaptic NMDA receptors through binding to the C-terminal tail "ESDV", and may be involved in the organization and trafficking of this population of receptors [51]. Intriguingly, among the four potential ligands of SjGIPC3 identified in this study, one is the glutamate receptor NMDA, which also bears a class I motif "TTTL"; thus, we cannot rule out the possibility that SjGIPC3 may also have a physiological function in the regulation of NMDA receptor trafficking, similar to its orthologue in mouse.

\section{Conclusions}

For the first time, we have presented the molecular characterization of the PDZ domain-containing protein
GIPC3 from S. japonicum. We defined the ligand binding specificity of the SjGIPC3-PDZ domain by screening a random peptide library constructed with human genomic DNA. The ligand binding ability of the SjGIPC3PDZ domain is relatively extensive; it can bind both type I and type II ligand motifs, which hints at its potential to play multiple roles during the development of S. japonicum, especially in the adult stage. Our study also provided a practical method for predicting potential ligands of SjGIPC3. Ongoing studies to identify more ligands involved in signal transduction followed by improvement of the quality of the S. japonicum proteomic dataset will assist in the discovery of novel drug targets against the parasite.

\section{Ethics statement}

All procedures performed on animals within this study were conducted following animal husbandry guidelines of the Chinese Academy of Medical Sciences and with permission from the Experimental Animal Committee.

\section{Additional file}

Additional file 1: Table S1. Primer sequences used in this study. This file contains the information about the primers used for qRT-PCR and molecular cloning.

\section{Competing interests}

The authors declare that they have no competing interests.

\section{Authors' contributions}

YM, PC, and YG conceived and designed the experiments. YM, HH, SL, and PC performed the experiments. YM, PC, and YG analyzed the data. YM, PC, and $Y G$ wrote the manuscript. All authors read and approved the final version of the manuscript.

\section{Acknowledgments}

This work was supported by the National Basic Research Program of China (2012CB517606, 2013CB530805, 2011CB964901), the National High

Technology Research and Development Program of China (2011AA020116), Program for Changjiang Scholars and Innovative Research Team in University-PCSIRT (IRT0909), and 111 Project (B08007). 


\section{Author details}

'National Key Laboratory of Medical Molecular Biology, Dept. of Physiology and Pathophysiology, School of Basic Medicine, Peking Union Medical College, Institute of Basic Medical Sciences, Chinese Academy of Medical Sciences, Beijing 100005, P.R. China. ${ }^{2}$ MOH Key Laboratory of Systems Biology of Pathogens, Institute of Pathogen Biology, Chinese Academy of Medical Sciences \& Peking Union Medical College, Beijing 100730, P.R. China. ${ }^{3}$ Donnelly Centre for Cellular and Biomolecular Research, University of Toronto, Toronto, ON, Canada.

Received: 4 September 2012 Accepted: 5 October 2012

Published: 10 October 2012

\section{References}

1. Ismail M, Botros S, Metwally A, William S, Farghally A, Tao LF, Day TA Bennett JL: Resistance to praziquantel: direct evidence from Schistosoma mansoni isolated from Egyptian villagers. Am J Trop Med Hyg 1999, 60:932-935

2. Fenwick A, Rollinson D, Southgate V: Implementation of human schistosomiasis control: Challenges and prospects. Adv Parasitol 2006, 61:567-622.

3. Liu R, Dong HF, Guo Y, Zhao QP, Jiang MS: Efficacy of praziquantel and artemisinin derivatives for the treatment and prevention of human schistosomiasis: a systematic review and meta-analysis. Parasit Vectors 2011, 4:201.

4. Zhou Y, Zheng HJ, Chen YY, Zhang L, Wang K, Gou J, Huang Z: The Schistosoma japonicum genome reveals features of host-parasite interplay. Nature 2009, 460:345-351.

5. Berriman M, Haas BJ, LoVerde PT, Wilson RA, Dillon GP, Cerqueira GC, Mashiyama ST, Al-Lazikani B, Andrade LF, Ashton PD, et al: The genome of the blood fluke Schistosoma mansoni. Nature 2009, 460:352-358.

6. Young ND, Jex AR, Li B, Liu S, Yang L, Xiong Z, Li Y, Cantacessi C, Hall RS, Xu $X$, et al: Whole-genome sequence of Schistosoma haematobium. Nat Genet 2012, 44:221-225.

7. Archakov Al, Govorun VM, Dubanov AV, Ivanov YD, Veselovsky AV, Lewi P, Janssen P: Protein-protein interactions as a target for drugs in proteomics. Proteomics 2003, 3:380-391.

8. Dev KK: Making protein interactions druggable: targeting PDZ domains. Nat Rev Drug Discov 2004, 3:1047-1056.

9. Zhou L, Li F, Xu HB, Luo CX, Wu HY, Zhu MM, Lu W, Ji X, Zhou QG, Zhu DY: Treatment of cerebral ischemia by disrupting ischemia-induced interaction of nNOS with PSD-95. Nat Med 2010, 16:1439-1443.

10. Lee $H J$, Zheng JJ: PDZ domains and their binding partners: structure, specificity, and modification. Cell Commun Signal 2010, 8:8

11. Kalyoncu S, Keskin O, Gursoy A: Interaction prediction and classification of PDZ domains. BMC Bioinformatics 2010, 11:357.

12. Schlieker C, Mogk A, Bukau B: A PDZ switch for a cellular stress response. Cell 2004, 117:417-419.

13. De Vries L, Lou X, Zhao G, Zheng B, Farquhar MG: GIPC, a PDZ domain containing protein, interacts specifically with the $C$ terminus of RGSGAIP. Proc Natl Acad Sci USA 1998, 95:12340-12345.

14. Rousset $R$, Fabre $S$, Desbois $C$, Bantignies F, Jalinot $P$ : The $C$-terminus of the HTLV-1 Tax oncoprotein mediates interaction with the PDZ domain of cellular proteins. Oncogene 1998, 16:643-654.

15. Wang $L H$, Kalb RG, Strittmatter SM: A PDZ protein regulates the distribution of the transmembrane semaphorin, M-SemF. J Biol Chem 1999, 274:14137-14146.

16. Bunn $R C$, Jensen MA, Reed BC: Protein interactions with the glucose transporter binding protein GLUT1CBP that provide a link between GLUT1 and the cytoskeleton. Mol Biol Cell 1999, 10:819-832.

17. Wang L, Dutta SK, Kojima T, XU X, Khosravi-Far R, Ekker SC, Mukhopadhyay D: Neuropilin-1 modulates p53/caspases axis to promote endothelial cell survival. PLoS One 2007, 2:e1161.

18. Gao Y, Li M, Chen W, Simons M: Synectin, syndecan-4 cytoplasmic domain binding PDZ protein, inhibits cell migration. J Cell Physiol 2000, 184:373-379.

19. Tani TT, Mercurio AM: PDZ interaction sites in integrin alpha subunits. T14853, TIP/GIPC binds to a type I recognition sequence in alpha $6 \mathrm{~A} / \mathrm{alpha}$ 5 and a novel sequence in alpha 6B. J Biol Chem 2001, 276:36535-36542.

20. Blobe GC, Liu X, Fang SJ, How T, Lodish HF: A novel mechanism for regulating transforming growth factor beta (TGF-beta) signaling.
Functional modulation of type III TGF-beta receptor expression through interaction with the PDZ domain protein, GIPC. J Biol Chem 2001, 276:39608-39617.

21. Ligensa T, Krauss S, Demuth D, Schumacher R, Camonis J, Jaques $G$ Weidner KM: A PDZ domain protein interacts with the C-terminal tail of the insulin-like growth factor-1 receptor but not with the insulin receptor. J Biol Chem 2001, 276:33419-33427.

22. Wu J, O'Donnell M, Gitler AD, Klein PS: Kermit 2/XGIPC, an IGF1 receptor interacting protein, is required for IGF signaling in Xenopus eye development. Development 2006, 133:3651-3660.

23. Jeanneteau F, Diaz J, Sokoloff P, Griffon N: Interactions of GIPC with dopamine D2, D3 but not D4 receptors define a novel mode of regulation of $\mathrm{G}$ protein-coupled receptors. Mol Biol Cell 2004, 15:696-705.

24. Hirakawa T, Galet C, Kishi M, Ascoli M: GIPC binds to the human lutropin receptor (hLHR) through an unusual PDZ domain binding motif, and it regulates the sorting of the internalized human choriogonadotropin and the density of cell surface hLHR. J Biol Chem 2003, 278:49348-49357.

25. Hu LA, Chen W, Martin NP, Whalen EJ, Premont RT, Lefkowitz RJ: GIPC interacts with the beta1-adrenergic receptor and regulates beta1adrenergic receptor-mediated ERK activation. J Biol Chem 2003, 278:26295-26301.

26. Bohlson SS, Zhang M, Ortiz CE, Tenner AJ: CD93 interacts with the PDZ domain-containing adaptor protein GIPC: implications in the modulation of phagocytosis. J Leukoc Biol 2005, 77:80-89.

27. Tan C, Deardorff MA, Saint-Jeannet JP, Yang J, Arzoumanian A, Klein PS: Kermit, a frizzled interacting protein, regulates frizzled 3 signaling in neural crest development. Development 2001, 128:3665-3674.

28. Katoh M: GIPC gene family (Review). Int I Mol Med 2002, 9:585-589.

29. Naccache $S N$, Hasson T, Horowitz A: Binding of internalized receptors to the PDZ domain of GIPC/synectin recruits myosin VI to endocytic vesicles. Proc Natl Acad Sci USA 2006, 103:12735-12740.

30. Djiane A, Mlodzik M: The Drosophila GIPC homologue can modulate myosin based processes and planar cell polarity but is not essential for development. PLoS One 2010, 5:e11228.

31. Muders MH, Vohra PK, Dutta SK, Wang E, Ikeda Y, Wang L, Udugamasooriya DG, Memic A, Rupasinghe CN, Baretton GB, et al: Targeting GIPC/synectin in pancreatic cancer inhibits tumor growth. Clin Cancer Res 2009, 15:4095-4103.

32. Chittenden TW, Pak J, Rubio R, Cheng H, Holton K, Prendergast N, Glinskii V, Cai Y, Culhane A, Bentink S, et al: Therapeutic implications of GIPC1 silencing in cancer. PLoS One 2010, 5:e15581.

33. Patra CR, Rupasinghe CN, Dutta SK, Bhattacharya S, Wang E, Spaller MR, Mukhopadhyay D: Chemically modified peptides targeting the PDZ domain of GIPC as a therapeutic approach for cancer. ACS Chem Biol 2012, 7:770-779.

34. Cai P, Bu L, Wang J, Wang Z, Zhong X, Wang H: Molecular characterization of Schistosoma japonicum tegument protein tetraspanin-2: sequence variation and possible implications for immune evasion. Biochem Biophys Res Commun 2008, 372:197-202.

35. Marchler-Bauer A, Lu S, Anderson JB, Chitsaz F, Derbyshire MK, DeWeeseScott C, Fong JH, Geer LY, Geer RC, Gonzales NR, et al: CDD: a Conserved Domain Database for the functional annotation of proteins. Nucleic Acids Res 2011, 39:D225-229.

36. Kim DE, Chivian D, Baker D: Protein structure prediction and analysis using the Robetta server. Nucleic Acids Res 2004, 32:W526-531.

37. Wu W, Cai P, Chen Q, Wang H: Identification of novel antigens within the Schistosoma japonicum tetraspanin family based on molecular characterization. Acta Trop 2011, 117:216-224.

38. Cai P, Piao X, Hou N, Liu S, Wang H, Chen Q: Identification and characterization of Argonaute protein, Ago2 and its associated small RNAs in Schistosoma japonicum. PLoS Negl Trop Dis 2012, 6:e1745.

39. Huang $\mathrm{H}$, Gao Y: A method for generation of arbitrary peptide libraries using genomic DNA. Mol Biotechnol 2005, 30:135-142.

40. Song E, Gao S, Tian R, Ma S, Huang H, Guo J, Li Y, Zhang L, Gao Y: A high efficiency strategy for binding property characterization of peptidebinding domains. Mol Cell Proteomics 2006, 5:1368-1381.

41. Huang HM, Zhang L, Cui QH, Jiang TZ, Ma SC, Gao YH: Finding potential ligands for PDZ domains by tailfit, a JAVA program. Chin Med Sci J 2004, 19:97-104. 
42. Liu S, Cai P, Hou N, Piao X, Wang H, Hung T, Chen Q: Genome-wide identification and characterization of a panel of house-keeping genes in Schistosoma japonicum. Mol Biochem Parasitol 2012, 182:75-82.

43. Reed BC, Cefalu C, Bellaire BH, Cardelli JA, Louis T, Salamon J, Bloecher MA, Bunn RC: GLUT1CBP(TIP2/GIPC1) interactions with GLUT1 and myosin VI: evidence supporting an adapter function for GLUT1CBP. Mol Biol Cell 2005, 16:4183-4201.

44. Finan D, Hartman MA, Spudich JA: Proteomics approach to study the functions of Drosophila myosin VI through identification of multiple cargo-binding proteins. Proc Natl Acad Sci USA 2011, 108:5566-5571.

45. Kim J, Kim I, Yang JS, Shin YE, Hwang J, Park S, Choi YS, Kim S: Rewiring of PDZ domain-ligand interaction network contributed to eukaryotic evolution. PLoS Genet 2012, 8:e1002510

46. Collins JJ 3rd, King RS, Cogswell A, Williams DL, Newmark PA: An atlas for Schistosoma mansoni organs and life-cycle stages using cell type-specific markers and confocal microscopy. PLOS Negl Trop Dis 2011, 5:e1009.

47. Piao X, Cai P, Liu S, Hou N, Hao L, Yang F, Wang H, Wang J, Jin Q, Chen Q: Global expression analysis revealed novel gender-specific gene expression features in the blood fluke parasite Schistosoma japonicum. PLoS One 2011, 6:e18267.

48. Charizopoulou N, Lelli A, Schraders M, Ray K, Hildebrand MS, Ramesh A, Srisailapathy CR, Oostrik J, Admiraal RJ, Neely HR, et al: Gipc3 mutations associated with audiogenic seizures and sensorineural hearing loss in mouse and human. Nat Commun 2011, 2:201.

49. Rehman AU, Gul K, Morell RJ, Lee K, Ahmed ZM, Riazuddin S, Ali RA, Shahzad M, Jaleel AU, Andrade PB, et al: Mutations of GIPC3 cause nonsyndromic hearing loss DFNB72 but not DFNB81 that also maps to chromosome 19p. Hum Genet 2011, 130:759-765.

50. Sirmaci A, Edwards YJ, Akay H, Tekin M: Challenges in whole exome sequencing: an example from hereditary deafness. PLoS One 2012, 7: e32000

51. Yi Z, Petralia RS, Fu Z, Swanwick CC, Wang YX, Prybylowski K, Sans N, Vicini S, Wenthold RJ: The role of the PDZ protein GIPC in regulating NMDA receptor trafficking. J Neurosci 2007, 27:11663-11675.

doi:10.1186/1756-3305-5-227

Cite this article as: Mu et al:: Molecular characterization and ligand binding specificity of the PDZ domain-containing protein GIPC3 from Schistosoma japonicum. Parasites \& Vectors 2012 5:227.

\section{Submit your next manuscript to BioMed Central and take full advantage of:}

- Convenient online submission

- Thorough peer review

- No space constraints or color figure charges

- Immediate publication on acceptance

- Inclusion in PubMed, CAS, Scopus and Google Scholar

- Research which is freely available for redistribution 